\title{
Effect dyslexia on early learning of mathematics to the children
}

\author{
Khalid Mohammed Almahrag (Corresponding author) \\ Special Education, King Saud University \\ 3749 Riyadh 12372 - 7959, Kingdom of Saudi Arabia \\ E-mail: almahrej@yahoo.com
}

\begin{abstract}
Many dyslexic children have problems in math, particularly the multiplication tables, fractions, decimals, percentages, ratios, and statistics. A dyslexic student usually requires extra teaching, mainly as new concepts do introduce. With this subject, it is vital to grasp each concept thoroughly before moving on. Although research has indicated that difficulties in language experienced by dyslexic people frequently go hand in hand with problems in mathematics, there has been too little emphasis on expert study for those having problems with math (Henderson, 2013). With the help of specialist teaching and the introduction of effective strategies into their learning, these people can get higher levels of mathematical understanding and functioning. This paper will examine how dyslexia can affect mathematics teaching and how cognitive weakness can affect mathematics teaching for dyslexic learners. Then explore how to assess and diagnose the mathematics difficulties for students with dyslexia. The researcher will also consider how to facilitate mathematical learning in children with dyslexia.
\end{abstract}

Keywords: Dyslexia, Mathematics, Early identification

DOI: $10.7176 / \mathrm{ADS} / 95-05$

Publication date:October $30^{\text {th }} 2021$

\section{Introduction}

The children's dyslexia has specific writing and spelling problems and sometimes a particular problem in mathematics included (British Dyslexia Association, 2002). Traditionally, dyslexia has concentrated mainly on literacy - the learning of the reading and spelling processes. However, some dyslexic difficulties also transmit into the teaching of mathematics. Based on initial studies, research results claim that about $60 \%$ of people with dyslexia have some problems with school math (Chinn \& Ashcroft, 2017). Of the $40 \%$ of people with dyslexia who do not seem to have math difficulties, about $11 \%$ of people with dyslexia do well in mathematics. The rest (29\%) also do children the same age, who have no learning difficulties. Surveys say that between 2-4 percent of people may have severe dyslexia, about one in each classroom - and more may have milder forms (Yeo, 2008). It is, therefore, significant that all teachers are aware of methods to help people with dyslexia.

The International Dyslexia Association characterizes dyslexia as a learning disability distinguished by expressive or receptive, oral, or written language difficulties. Problems may arise in reading, spelling, writing, speaking, or listening. Changes have recently occurred in the definitions of dyslexia. The difference is a return to the application of the word dyslexia. The term specific learning difficulty used to be equivalent to dyslexia. It is no longer the case as other specific learning difficulties have been defined, for instance, dyspraxia, attention deficit disorder (Riddick, 2012). Difficulties with mathematics do recognize as a possible result of dyslexia. Dyslexia likely causes difficulties in at least some fields of mathematics, most notably in numeracy.

Although dyslexia is a life condition, the influence on potential depends mainly on educational intervention. What may be very hard to discriminate is where an individual's difficulties with math are rooted. The learning of math is very dependent on teaching being appropriate to the person. People do not all learn identically, and, as math is a very sequential subject, each new idea builds on previous learning; failure can be cumulative. Riddick (2012) states that going back to mathematical themes may be essential to solving math problems.

\section{Areas that dyslexia learners have difficulties in mathematics}

Most people consider the rules of math's are consistent, some more consistent than the rules of spelling (Riddick, 2012). There are many inconsistencies in mathematics, which are often invisible. Math has more exclusion to the rules than many people believe (Riddick, 2012). These incompatibilities can significantly hinder the dyslexic child in that they challenge the security of learning. Many learners consider infractions problematic, we write $1 / 5$ $+3 / 5=4 / 5$, and the maximum numbers add. However, in $3 / 5 \times 2 / 5$, both top and bottom numbers are multiplied. We expect absolute exactness in answers and then hope students abandon this strict regime and estimate. In total 
numbers, the succession of words from left to right of the decimal points are units, tens, hundreds, and thousands. For decimals, the decimal point's succession from right to left is tenths, hundredths, thousandths.

There are some factors, which can affect the learning of mathematics. These may happen in seclusion or may interact to make a possible learning difficulty. Each person is an individual and will have a unique mixture of various levels of severity of these factors. With proper help, most of the problems connected with these factors can do lessened. and some areas that dyslexia children have difficulties in mathematics Language, Short Term, Direction Memory, Visual, Speed of Work, Sequencing, Anxiety, Conceptual Ability, Thinking Style, Notation and Understanding place value Mathematics has its vocabulary; for instance, algebra is only a math word. However, math has also shared words with other activities, so "take away" connects with food as well as subtraction (Riddick, 2012).

Innumeracy is when a series of words refer to the same math operation, such as add, more, and add. It is also possible to use more to imply subtraction, as in "Nick has three more pens than Ann." Nick has ten pens; how many cells does Ann have?" Math's word questions often use a unique mathematical form of the English language. It is not unexpected that people list word difficulties as one of the most challenging fields of math, even if they are not dyslexic (Miles, Haslum \& Wheeler, 2001).

Most dyslexic children have more short-term memory difficulties than non-Dyslexia (Chin \& Ashcroft, 2017). The results are that they can lose track in the middle of doing a multistep intellectual arithmetic problem or fail to realize a sequence of instructions. For instance, in adding $234+93$ in mind, starting with the units, there is $4+$ $3=7$, then the tens, $3+9=12$, carry on the 1 to end as $2+1=3$, then reverse these numbers $(7,2,3)$ to give 327. It is improbable that people with dyslexia can retain by rote learning basic number facts. Especially times table facts (Chin \& Ashcroft, 2017), or it may be that they cannot remember the succession of steps needed to finish a long division sum, particularly if the process has no sense or logic to confirm memory.

Learners tend to look for logicality (and thus patterns, rules, and connections) in what they learn. There are much more inconsistencies in the direction in mathematics than many people understand, for example. Any inconsistency can affect an uncertain learner, and many math learners are insecure. The difficulty recognizing differences between symbols, such as + and $\mathrm{x}$; or + and $\mathrm{ч}$, or the plan of work on the book page can cause problems, for example, if the distance between samples is very close. One of the odd things about math is the need to do it quickly. This requirement tends to increase disquiet and thus decrease precision. People with dyslexia usually are slower in maths, with many factors furthering this, such as slow recall of basic facts. A classroom study (Chinn \& Ashcroft, 2017) found that dyslexic pupils took about $50 \%$ more time to finish a set of arithmetic questions than their non-dyslexic peers. It is difficult for dyslexic children to concentrate on high numbers, and this highly slows their work. It is a perfect example of how choosing proper work techniques with dyslexic children, simple awareness, and adjustment can reduce stress and help them learn.

Math needs sequencing capabilities; This problem can connect with language problems in questions such as 'Take 17 away from 36," which presents the numbers in the reverse sequence for calculation, to "26 minus 16 ," which shows the numbers in the series in which the subtraction does compute. The succession of negative numbers can be bewildering as in negative coordinates $(-3,-6)$.

Most learners can have anxiety with mathematics (and some teachers) (Henderson \& Miles, 2001). For example, fractions often are the reason for disorder. It is a feature of people with dyslexia (and probably other learners, too) that if they pre-judge a question as too challenging to solve, they escape failure by not trying to solve the question. It is the hidden factor that has a connection with the above. The dyslexic pupils demonstrate a much higher proportion of "no attempts" in the arithmetic test. They determine they cannot get the question accurate, so they avoid failure by not trying the question. Learning needs the learner to take a risk and get inveigled in the learning process. Dread of default can stop the learner from taking the risk necessary for learning.

There is just a usual spread of conceptual ability in maths for people with dyslexia, and they can reach or above this potential with proper teaching and motivation (Henderson \& Miles, 2001). Another significant factor is that an idea's early misconception and initial wrong practice may create a dominant memory. For example, if pupils write 51 for 15 when they first meet this number, that will be a dominant memory unless the correction comes immediately. It is essential to make sure that each new idea does practice accurately.

Dyslexic strengths and difficulties share the same source - the dyslexic thinking style. People with dyslexia tend to think initially through pictures and images rather than through the internal monologue used by verbal thinkers (Yeo,2008). Nowadays, researchers have identified two thinking styles (Yeo, 2008). One style is a pattern and sequential, and the other is intuitional and entire. Most learners lie somewhere on a continuum between the two extremes of style, and indeed, this mixture is likely to be the most successful style as success in math tends to 
need flexibility in thinking. Understanding a learner's thinking style can be one of the essential pieces in the jigsaw of realizing the learner. Each style needs sub-skills, but this does not mean that the learner chooses to use the style more appropriate to his sub-skills.

The fractional and decimal notation may also prove confusion.

Some people with dyslexia do not readily understand the idea of place value, especially when there are zeros in a number $(20,040)$. They may also take longer to understand multiplying patterns and dividing by $10,100,1,000$ Etc.

\section{Effect cognitive weakness on mathematics learning for children with}

dyslexia, a significant proportion of dyslexic students have difficulty learning math (Miles \& Miles, 2004). Some dyslexic students' numeracy abilities do hinder by surface issues, such as difficulty recalling oral math facts and multiplication tables. Others have more severe problems like essential counting development is delayed. Students learn to repeat oral counting sequences later than their peers and control the demands of enumerating specific objects later (Chin \& Ashcroft, 2017). These students also seem to understand the main principle later than other students. On the other hand, however, as their counting skills evolve, dyslexic children with critical fundamental math difficulties do not seem to develop basic "number sense." In other words, they fail to create a "feel" for numerosities and a "feel" for abstract numbers (Henderson \& Miles, 2001).

In addition, we know that individuals with dyslexia may have difficulties with the language of mathematics and the concepts connected with it. These include spatial and numerical relations such as before, after, between, one more than, and one less. Some math terms like numerator and denominator, prime numbers, and prime factors, and carrying and borrowing may also be complex (Miles, Haslum \& Wheeler 2001).

Children may be bewildered by implicit, multiple senses of words, e.g., two as the name of a part in a sequence and a set of two objects. Difficulties may also happen around the concept of place value and the role of zero. Resolving word problems may be particularly challenging because of decoding, comprehension, sequencing, and understanding mathematical ideas. In understanding the complex nature of Dyslexia, Ansara (1996) made three general assumptions about learning, especially for individuals with dyslexia. These assumptions affect the way one needs to provide instruction. They are, learning involves the understanding of patterns. They are learning more difficult for dyslexic children than the others because of deficits that intervene with the ready recognition of patterns. The organization of units into wholes is complex for some dyslexic children due to a disability in handling spatial and temporary relationships or only problems with integration, sequencing, or memory.

Some learners are greatly intuitive in the way they learn and do maths. For example, if asked to find three consecutive numbers, which add up to 33 , they will divide 33 by three, arrive at 11 , and quickly conclude the trio with 10 and 12. Other learners are pattern and step by step in their style. They would approach the 33 questions algebraically, probably by inferring the equation $x(x+1)(x+2)=33$, which resolves to $x=10($ Chin \& Ashcroft, 2017). Learners require drawing on both these learning styles. An over-dependence on one style is in some way a disadvantage. People with dyslexia will often choose the style they consider the most reliable, the formulaic style, even though it may not be the best way to solve the difficulties.

\section{Assessment for diagnoses of the mathematic troubles for dyslexic leaner}

There are at least two ways a student may do identified for assessment (Chin \& Ashcroft, 2017). The first is that the education institution recognizes a learning or behavior problem and asks the student's parents to assess the student individually. The teacher must identify if the child has scores too far below their peers and present tests in a particular grade. Alternatively, the student's classroom teacher may identify some problem - possibly the student's work is below anticipations for their status or age. The student's behavior is shattering learning - and so the teacher directs the student for assessment.

The student's parents can also call or write to the school or the director of special education and ask their child to evaluate. They may suspect that the child is not progressing as they should be or notice problems in how the child learns. If the institution suspects that the child may have a disability, it must conduct an assessment (Riddick, 2012). The SEN Code of Practice (2002) requires schools to provide appropriate support so that all dyslexic children can benefit from an education (Karp \& Howell 2004). In line with the Code of Practice, the SENCO will do made an Individual Education Plan, setting out the school's steps to provide appropriate support for the child's needs.

If the local education authority does not think that the child has a disability, they may refuse to assess them. 
However, they must appraise the parents in writing about their reasons for refusing. Suppose parents feel mighty that their child does, indeed, have a disability that needs individual education. In that case, they may request a due process hearing, where they will have the opportunity to show why they think their child should do evaluated.

While all students are different in many ways, they may also share something in common. Each may be a student who has dyslexia that will require special education services in the school setting. Before deciding about special education services, each student will require an evaluation conducted by specially trained educational personnel, combining a school psychologist. A speech/language pathologist, special education and standard education teachers, social workers, and, when appropriate, medical personnel.

Assessment in educational settings serves five fundamental purposes (Miles, Haslum \& Wheeler, 2001): Screening and identification. To screen children and identify those who may be experiencing protraction or learning difficulties. Eligibility and diagnosis. To decide whether a child has a disability and is eligible for special education services and to diagnose the peculiar nature of the student's problems or disability. IEP development and placement. To present detailed information so that an Individualized Education Program (IEP) may evolve, and appropriate decisions may do made about the child's education. Educational planning. To develop and design instruction appropriate to the child's unique needs. Evaluation. To evaluate students' progress.

A major issue seems to be identifying dyslexia across the population. There are problems within the school system of proper and early identification. A comprehensive screening system for dyslexia within higher education would be a good idea, although some higher education institutions already use this. For testing for numeracy difficulties, it is not a bad idea to create an informal diagnosis. Think about what the child needs to know, combining the prerequisite knowledge, and build the test accordingly. One of the most revealing diagnostic questions is, "How did you do that? Talk me through your work." Moreover, remember that the mistakes are more revealing than the correct answers. The end outcome of a mathematics test should be a lot more than just a number.

There is a test for dyscalculia, written by Professor Brian Butterworth (1987). He devised DfES (the Department for Education and Skills) tests that involve a sequence of simple math questions, combining counting dots on a computer screen, or comparing two sets of images and indicating the larger. Children are classed according to their time to answer the questions, with different response times expected for other groups. Butterworth's test for dyscalculia will deal with early identification. Some children may then advance beyond the levels of concern. Early indicators will be problems dealing with sequences, prolonged retention of basic facts, no sense of number, an inability to see prototypes in information.

Specific difficulties, for instance, reading and comprehending the unique language and vocabulary of mathematics, may "click in" after a comparatively successful start in the subject. A student may excel at mental arithmetic and fail when needed to document (or vice versa). Different fields of mathematics may well produce different reactions from other students. It is helpful to analyze a mathematics task by considering vocabulary, basic fact knowledge, realizing the four operations, memory (short and long term), sequencing ability, generalizing, documenting, and spatial awareness. And then identify which area makes a problem for the learner (Miles, Haslum \& Wheeler 2001). A primary indicator of math's disability is when the student will not show expectations in studies with no apparent reason, such as an emotional state or an illness.

Underachievement may manifest in specific ways, such as difficulties understanding the value or worth of numbers or having difficulty realizing that eight is one less than 9 (Karp \& Howell 2004). The NNS requires, for example, recalling basic number facts - or, possibly, using algorithms (procedures) without understanding the reasoning behind them or how to calculate answers (Karp \& Howell 2004). Some students with good memories and good general abilities may not present as underachievers within a class. However, they may be dramatically underachieving in their true potential. Some students falter in the counting-on phase of development. The (part) question of how dyscalculia differs from 'dyslexia with numbers' will depend on the explanation of "dyslexia with numbers. "A primary part of the assessment process often involves examining a student's work, either by choosing work samples that can be analyzed to identify academic skills and deficits or by conducting a portfolio assessment, where folders of the student's work investigate (Karp \& Howell 2004).

When collecting work samples, the teacher chooses work from the areas where the student has difficulty and regularly examines them. The teacher might identify such elements as to how the student does instruct to do the activity (e.g., orally, in writing), how long it took the student to finish the training, the pattern of mistakes (e.g., reversals when writing, Etc.), and the pattern of correct answers. Analyzing the student's work in this way can 
yield valuable insight into the nature of their difficulties and advise probable solutions. Sustaining portfolios of student work has become a popular way for teachers to track student success. Teachers can monitor a student's progress, identify problems that recur, determine whether concepts are understood or not understood, and determine if skills are being developed by collecting student work in one place. The portfolio can do analyzed in much the same way as choice work samples. It can establish the basis for discussions with the student or other teachers about difficulties and successes and determine what modifications teachers might make.

\section{Discussion}

The proper use of calculators can be beneficial. As with every piece of equipment, there can be disadvantages and cautions. The calculator can be used to carry out sums that would take a long time on paper, for example, $522 \div 11.72$. Calculators are also a source of information in advance provided by log tables and slide rules. They can also compute hard statistical data and present data graphically. For people with dyslexia, there needs to be some attention over right keys and correct order of keys, verified by pre-estimations (Yeo, 2008). So if we ask for "5.3 divided into 607 ," we key in the numbers in reverse order $607 \div 5.3$, seeking an answer just over 100 . If the keys are used without error and in the correct order, the calculator can show up or develop patterns. Software should be able to offer the dyslexic learner a practical learning input. Voice output, graphics, symbols, and text can all do included to provide a multisensory experience to the learner, and, where appropriate, a diagnostic element can be incorporated. Notwithstanding that some programs are near to this, most are not.

Existing programs fall into three groups: Games that current practice (Perfect Times - online games to practice the multiplication tables). The biggest problem with these is that the appearance and design can be somewhat age peculiar to the math's level in a similar way that books with a reading level appropriate for poor readers are often far too young. Some have built-in advanced math construction, which enables the user to target the required level of math. Sometimes the games can be frustrating in that they slow up the math or take immortality to complete and achieve the goal. It is possible to accommodate dyslexic children with poor presentation skills and a slow production rate with Excel and plot graphs programs. Some programs are just books on screen, making use of the potential of the computer to do so much more than show print (Language Shock - Dyslexia across cultures).

Nevertheless, for a dyslexic learner, these programs may be easier to trail through than a book. The child can read E-Books on ordinary computers, PDAs, and some unique e-book readers. A large screen is more comfortable and precise than printed text. A dyslexic child may also be able to listen to the text. DAISY format can provide e-books which the student can listen to but also search and move around. The software can allow the learner to work independently. The success of this depends on the design of the program. It is essential, for example, that the learner can navigate the program quickly. It is good if there is a clear voice output and that the program uses good images. Many people with dyslexia consider view on the screen overdone. Some designers seem to strive to put everything from their design collection onto the screen. Preparing the proper balance is tricky as learners are very individual.

Multiplication and division are the hardest for the child to master. It will make it easier for the person with dyslexia to learn if they understand the concept. The following may assist take five pairs of items, gloves, socks, shoes, toy animals, anything as long as the pairs are identical. Layout the teams before the child, demonstrate to her that there are two items in each pair, one pair has two items, two couples have four items, Etc. (Miles 1992). When the child can see the five teams have ten things, clarify as they write it down what $5 \times 10$ signifies. This exercise can be reiterated with each pair until they understand what the "2 times" implies. When the child is familiar with the two times table, they should start to work on all the tables in the following order: $2 \mathrm{x}, 10 \mathrm{x}, 11 \mathrm{x}$, $5 x, 3 x, 4 x, 9 x, 6 x, 7 x, 8 x$, then eventually $12 x$, which they should learn from the other tables. When we first use worksheets, utilize pictures of known animals or items for students to count. If a student has a problem with one fact, we must show them how to use them to help them remember.

Games work particularly well with dyslexic children as they dislike basic worksheets (Riddick, 2012). Children like playing Bingo. This multiplication Bingo game is a big hit for most children (Henderson \& Miles 2001). Bingo type card can be made on a piece of paper with the answers to all the multiplication facts, up to $6 \times 6$ with regular dice, $9 \times 9$, and $12 \times 12$ (or with the polyhedral dice available at school supply stores). It must take turns to roll the dice, multiply the two rolled numbers, and mark them off on the players' Bingo sheets. Without making it too evident, let the child win some games to set up their self-confidence. We must teach the child to talk through math problems, saying it carefully without disturbing others. It will employ ear as well as visual memory abilities. Explain to them how this will assist because the brain can store different types of memories.

When teaching about money, it is better to use real coins instead of plastic; this is far more fun and exciting for 
the children (Karp \& Howell 2004). Children are excited as they do give real coins to sort. They make good progress in their knowledge of the values of the different currencies. It is not always essential to spend a fortune on items for tactile use; change, pieces of cereal, simple circles clipped out of colored paper are perfect for whole number and fraction work.

\section{Conclusion}

The dyslexic child should be given additional help in mathematics and self-esteem (Humphrey, 2001). Methods of helping a person with dyslexia depend on the level of their weaknesses. The teacher and other people helping people with dyslexia should understand dyslexia: what it is, the reasons for it, its indications, various types, and how it affects learning — knowing facts about dyslexia, a tutor or a supervisor will learn how to deal with it.

Its indicators are inability to calculate, poor memory, bad handwriting, reversals substitutions. However, there are different types of dyslexia when a person can have a good memory of figures but is terrible at calculating. One type deals well with the sequencing of operations and is accurate in detail. Those with good memory follow sequences and make math operations step-by-step (Chinn\& Ashcroft, 2017). Because of good memory, they can remember the formula, but they often do not understand what sense it has and what stands for the symbols. They are attentive to the details but do not see the big picture. Another type of dyslexic considers the big picture and understands math's patterns but cannot compute well and are bad at sequencing. They understand mathematical concepts. After solving problems mentally, they have difficulty verbalizing and explaining their answers. Knowing which weaknesses and dyslexia a child has, the teacher can facilitate working with everyone. Dyslexia cannot do cured, but teaching a person with dyslexia by facilitating techniques that tolerate his weaknesses can give a positive outcome.

There are specific requirements for math teachers tutoring people with dyslexia. It is not enough to be just a good mathematician and understand the nature of mathematics, which is apparent in his mind what stands behind all the symbols. Besides that, they must be good at explaining the material, and much patience is required. Dyslexia and its common indicators should be familiar to them. Teachers need to be capable of explaining ideas from different angles over and over again. They should have no prejudices towards people with dyslexia. Dyslexia is not the result of a lack of intelligence.

\section{References}

British Dyslexia Association. (2002). The dyslexia handbook 2002. London: Author

Chinn, S., \& Ashcroft, R. E. (2017). Mathematics for dyslexics and dyscalculics: a teaching handbook. John Wiley \& Sons.

Henderson, A. (2013). Dyslexia, Dyscalculia, and Mathematics: A practical guide. Routledge.

Henderson, A. (1998) Maths for the Dyslexic: a practical guide. London: David Fulton Publishers.

Henderson, A., \& Miles, E. (2001). Introductory Topics in Mathematics for Dyslexia (Vol. 7). Wiley.

Humphrey, N. (2001a). Self-concept and self-esteem in developmental dyslexia: implications for teaching and learning. Liverpool John Moores University (United Kingdom).

Karp, K., \& Howell, P. (2004). Building responsibility for learning in students with special needs. Teaching children mathematics, 11(3), 118-126.

Mahon, D., Chinn, S., Van Elswijk, R., Harmsen, H., \& Kay, J. (1999). Comparative study of perceived difficulties in mathematics experienced by children with specific learning disabilities in Ireland, England, and The Netherlands. Irish Educational Studies, 18(1), 199-209.

Miles, T. R., Haslum, M. N., \& Wheeler, T. J. (2001). The mathematical abilities of dyslexic 10-yearolds. Annals of dyslexia, 51(1), 299-321.

Miles, T. R., \& Miles, E. (Eds.). (2004). Dyslexia and mathematics. Psychology Press.

Riddick, B. (2012). Living with dyslexia: The social and emotional consequences of specific learning difficulties/disabilities. Routledge.

Yeo, D. (2008). Dyslexia, dyspraxia, and mathematics. John Wiley \& Sons. 